\title{
EARLY-STAGE MASSIVE STAR FORMATION NEAR THE GALACTIC CENTER: Sgr C
}

\author{
S. Kendrew ${ }^{1,4}$, A. Ginsburg ${ }^{2}$, K. Johnston ${ }^{1}$, H. Beuther ${ }^{1}$, J. Bally ${ }^{2}$, C. J. Cyganowski ${ }^{3,5}$, And C. Battersby ${ }^{2}$ \\ ${ }^{1}$ Max-Planck-Institut für Astronomie, Königstuhl 17, D-69117 Heidelberg, Germany; kendrew@ mpia.de \\ ${ }^{2}$ CASA, University of Colorado at Boulder, UCB 389, Boulder, CO 80309, USA \\ ${ }^{3}$ Harvard-Smithsonian Center for Astrophysics, Cambridge, MA 02138, USA \\ Received 2013 June 21; accepted 2013 August 28; published 2013 September 19
}

\begin{abstract}
We present near-infrared spectroscopy and $1 \mathrm{~mm}$ line and continuum observations of a recently identified site of high mass star formation likely to be located in the Central Molecular Zone (CMZ) near Sgr C. Located on the outskirts of the massive evolved $\mathrm{H}$ II region associated with $\mathrm{Sgr} \mathrm{C}$, the area is characterized by an Extended Green Object (EGO) measuring $\sim 10^{\prime \prime}$ in size $(0.4 \mathrm{pc})$, whose observational characteristics suggest the presence of an embedded massive protostar driving an outflow. Our data confirm that early-stage star formation is taking place on the periphery of the Sgr C H II region, with detections of two protostellar cores and several knots of $\mathrm{H}_{2}$ and Brackett $\gamma$ emission alongside a previously detected compact radio source. We calculate the cores' joint mass to be $\sim 10^{3} M_{\odot}$, with column densities of $1-2 \times 10^{24} \mathrm{~cm}^{-2}$. We show the host molecular cloud to hold $\sim 10^{5} M_{\odot}$ of gas and dust with temperatures and column densities favorable for massive star formation to occur, however, there is no evidence of star formation outside of the EGO, indicating that the cloud is predominantly quiescent. Given its mass, density, and temperature, the cloud is comparable to other remarkable non-star-forming clouds such as G0.253 in the eastern CMZ.
\end{abstract}

Key words: infrared: ISM - ISM: jets and outflows - stars: formation

Online-only material: color figures

\section{INTRODUCTION}

The Central Molecular Zone (CMZ) of the Milky Way (MW) is a chemically and dynamically complex region containing up to $10 \%$ of the Galaxy's molecular gas, concentrated in dense and turbulent molecular clouds $(-2.5 \leqslant l \leqslant 3.5,|b| \leqslant 0.5)$. Densities and turbulent velocities are known to be approximately an order of magnitude higher than clouds in the MW disk (Morris \& Serabyn 1996), and shocks are widely observed (Riquelme et al. 2010). The CMZ presents an ideal laboratory for the study of star formation in extreme environments.

The distribution of molecular gas in the CMZ is asymmetric around the Galactic center (GC), with roughly two-thirds of the gas found at positive (eastern) longitudes; this is mirrored in the observed distribution of CMZ star formation (Yusef-Zadeh et al. 2009, YZ09 hereafter). The eastern CMZ is home to several star-forming regions, e.g., Sgr B2.

The only known star-forming region in the western $\mathrm{CMZ}$ is Sgr C (Figure 1). Lang et al. (2010) identify the region's main components as a $10 \mathrm{pc} \mathrm{HII} \mathrm{region} \mathrm{at}(l, b)=(359.43,-0.09$, assuming a distance of $8.5 \mathrm{kpc}$; Reid et al. 2009), accompanied by a distinctive non-thermal filament (Liszt \& Spiker 1995). The $\mathrm{H}$ II region contains more than $250 M_{\odot}$ of ionized gas, powered by at least one O4-O6 star (Liszt \& Spiker 1995; Odenwald \& Fazio 1984), however its stellar population remains poorly characterized. To the east of the $\mathrm{H}$ II region lies a pillar-shaped cloud, whose mass has been estimated at $10^{5} M_{\odot}$ (Lis et al. 1991; Lis \& Carlstrom 1994).

Molinari et al. (2011) recently proposed that Sgr C lies at the western vertex of a twisted elliptical ring of molecular gas and dust, $100 \times 60 \mathrm{pc}$ in size. The eastern longitude extremum is formed by Sgr B2. They propose that the collision of orbit

\footnotetext{
${ }_{4}$ Also at Department of Astrophysics, University of Oxford, Keble Road, Oxford OX1 3RH, UK.

5 NSF Astronomy and Astrophysics Postdoctoral Fellow.
}

systems at the extrema give rise to strong shocks, driving enhanced star formation. Given the prolific star formation rate of Sgr B2, Sgr C must be examined more closely to understand the observed asymmetry in the CMZ.

The first evidence of high mass star formation in the Sgr C cloud was reported by Forster \& Caswell (2000), who detected a faint $8-9 \mathrm{GHz}$ radio source measuring $0.06 \times 0.01 \mathrm{pc}$ near its tip. Using data from the Spitzer GALCEN survey (Stolovy et al. 2006), YZ09 identify a region of extended $4.5 \mu \mathrm{m}$ emission-a so-called Extended Green Object (EGO; Cyganowski et al. 2008) - within $\sim 5^{\prime \prime}$ from the radio source. Numerous studies have found EGOs to be strongly associated with early-stage high mass star formation and outflows (De Buizer \& Vacca 2010; Cyganowski et al. 2009). Three $\mathrm{CH}_{3} \mathrm{OH}$ masers are seen within the EGO at velocities consistent with the $\mathrm{Sgr} \mathrm{C}$ systemic velocity ( -55 to $-65 \mathrm{~km} \mathrm{~s}^{-1}$ ), supporting this scenario (Caswell 2009; Chambers et al. 2011). In this Letter, we present new infrared (IR) and millimeter data toward the EGO, which we refer to as G359.44-0.102, showing the presence of two massive protostellar cores and emission knots indicative of an outflow.

\section{DATA AND OBSERVATIONS}

TripleSpec near-infrared spectroscopy. To identify the origin of G359.44-0.102's IR emission, we carried out mediumresolution $\left(R \sim 100 \mathrm{~km} \mathrm{~s}^{-1}\right)$ spectroscopy in the near-infrared $K$-band, using TripleSpec on the $3.5 \mathrm{~m}$ ARC Telescope at Apache Point Observatory, in 2012 August (PI: Ginsburg). The $K$-band contains several $\mathrm{H}_{2}$ emission lines, most notably the $1-0 S(1)$ line at $2.12 \mu \mathrm{m}$, frequently observed in outflows from young stellar objects (Varricatt et al. 2010; Davis et al. 2010). Spectra were obtained at four positions with a 1".1 slit. The data were taken in ABBA slit-nodding mode with a series of $30 \mathrm{~s}$ exposures, for a total integration time of 2-6 minutes pointing ${ }^{-1}$.

Submillimeter Array (SMA). High-resolution data to search for dense star-forming gas toward the G359.44-0.102 EGO 

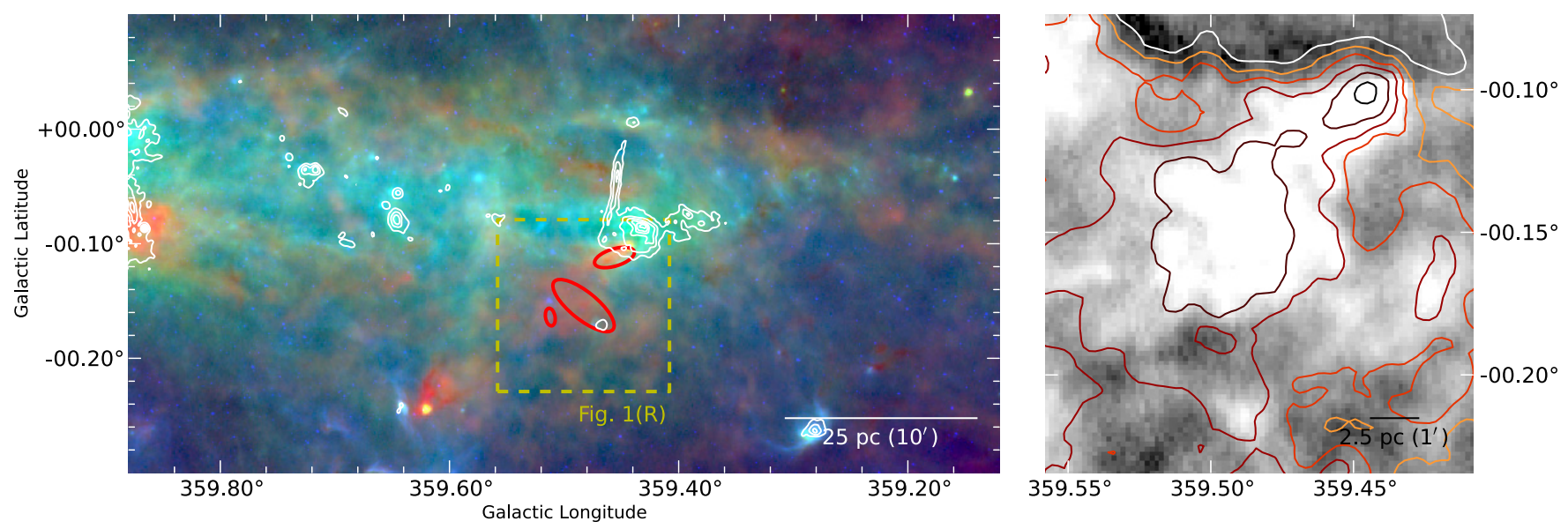

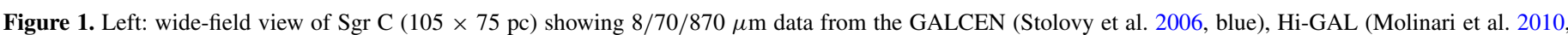

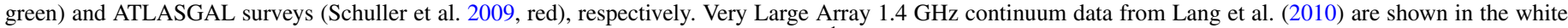

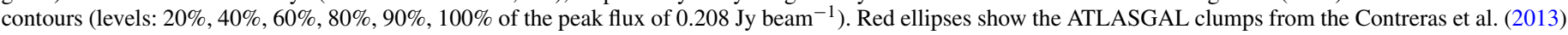

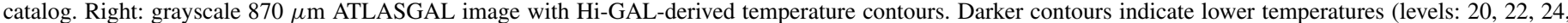
26, 28 and $30 \mathrm{~K}$ ).

(A color version of this figure is available in the online journal.)
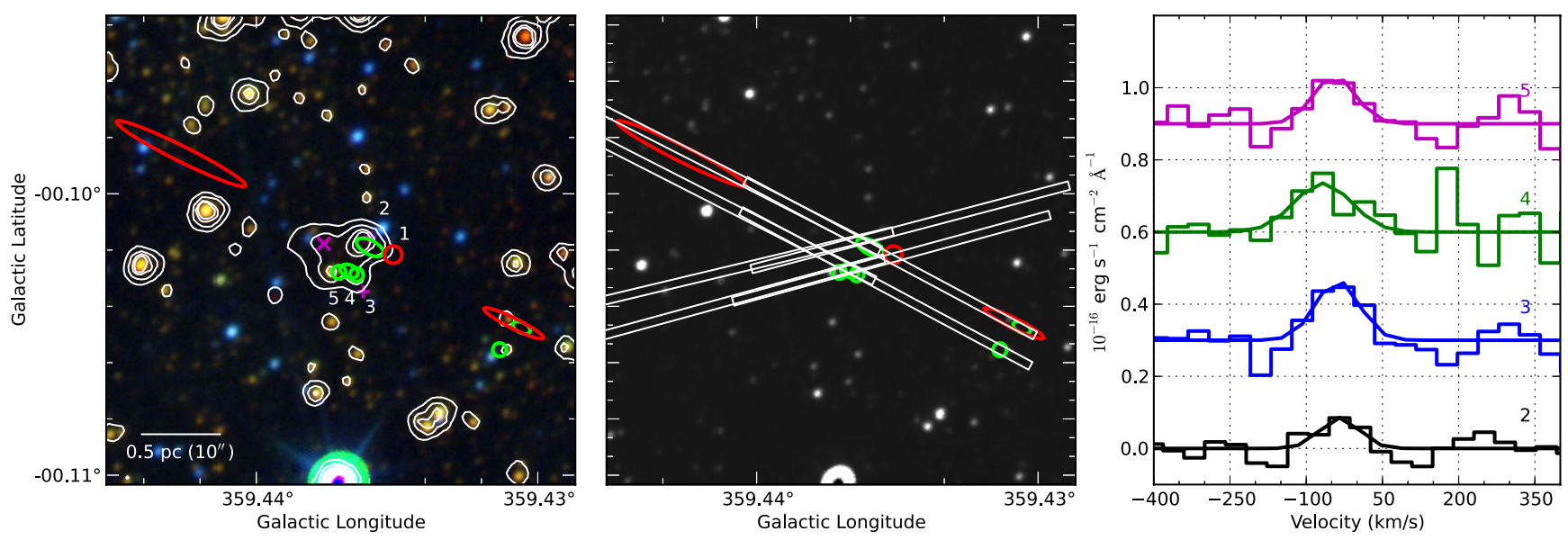

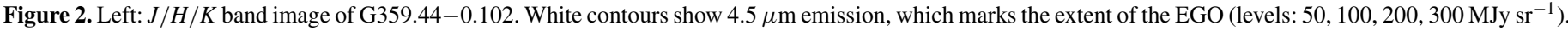

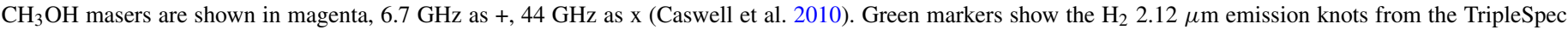

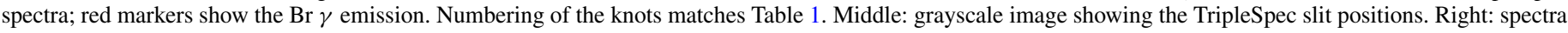
of the $\mathrm{H}_{2}$ lines.

(A color version of this figure is available in the online journal.)

were obtained in 2009 June with the compact-north configuration of SMA at $280 \mathrm{GHz}$ (PI: Kauffmann). These data consist of a mosaic of five $\sim 45^{\prime \prime}$ fields covering the EGO and its surrounding clump with spectral resolution of $0.44 \mathrm{~km} \mathrm{~s}^{-1}$. The observations covered the $\mathrm{N}_{2} \mathrm{H}^{+}$(3-2) line at $279.512 \mathrm{GHz}$, commonly used to trace dense gas, with rms noise of $0.23 \mathrm{Jy}$ beam $^{-1}$ when imaged at $2 \mathrm{~km} \mathrm{~s}^{-1}$ resolution. A combined self-calibrated continuum image was produced from the $2 \mathrm{GHz}$ sidebands centered at 278.8 and $288.8 \mathrm{GHz}$, with combined rms noise of $0.012 \mathrm{mJy}^{\text {beam }}{ }^{-1}$. The synthesized beam of the continuum and line images is $\sim 2^{\prime \prime}$. Data reduction and analysis were performed using CASA.

Survey data. The CMZ was covered by a number of Galactic Plane surveys. The Herschel Hi-GAL survey (Molinari et al. 2010) was used to derive temperature estimates toward the EGO and its host cloud, as described in Battersby et al. (2011). A Galactic cirrus emission model was estimated using an improved iterative technique which varies the source threshold value until it converges, classifying everything below that threshold as part of the diffuse cirrus. The emission model at each wavelength was subtracted from each of the five Hi-GAL bands, which were fit with a modified blackbody assuming $\beta=1.75$ to derive the dust temperature at each point (Figure $1 ; \beta$ derived from Ossenkopf \& Henning (1994) opacity law). The $870 \mu \mathrm{m}$ ATLASGAL survey (Schuller et al. 2009) traces cold dense gas at a spatial resolution of $\sim 19^{\prime \prime}$ (Figure 1). Fluxes at this wavelength were used to determine the physical properties of the EGO's host cloud (Contreras et al. 2013). Spectral line data at $3 \mathrm{~mm}$ from the Mopra CMZ survey (Jones et al. 2012) were used to determine the velocity of the EGO's region. Data on masers in the region were obtained from YZ09, Chambers et al. (2011) and Caswell (2009).

\section{RESULTS}

\subsection{TripleSpec Near-infrared Spectroscopy}

The near-infrared spectra reveal a number of emission locations toward the EGO and in its vicinity (Figure 2). Within the extent of the $4.5 \mu \mathrm{m}$ emission we find four knots of $\mathrm{H}_{2}$ emission 


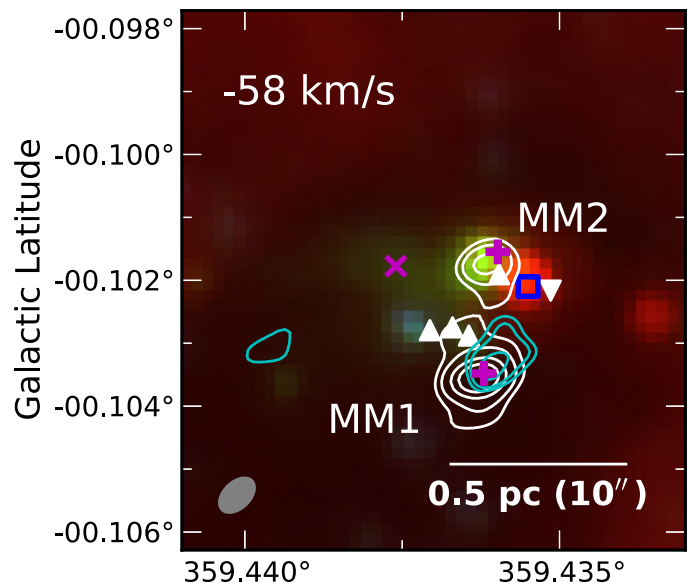

$359.440^{\circ}$ $359.435^{\circ}$

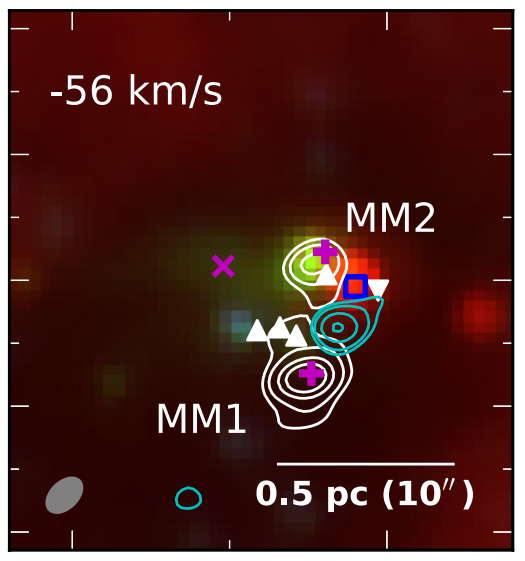

$359.440^{\circ}$ $359.435^{\circ}$

Galactic Longitude

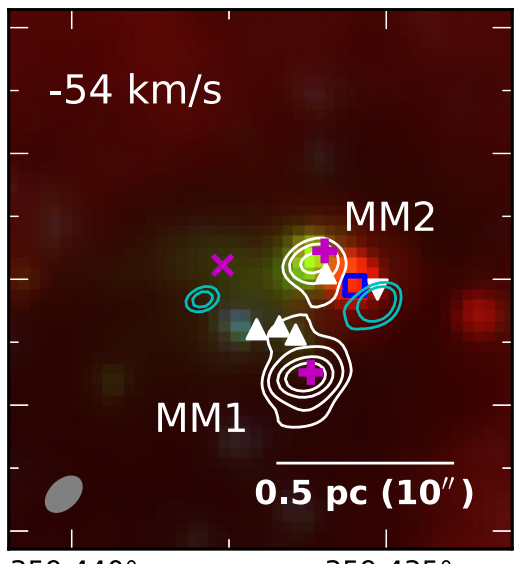

$359.440^{\circ}$ $359.435^{\circ}$

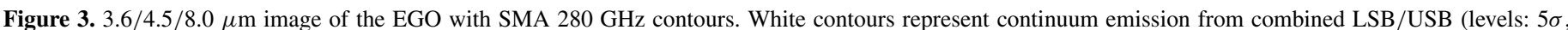

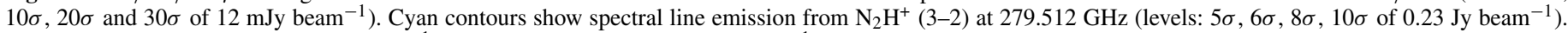

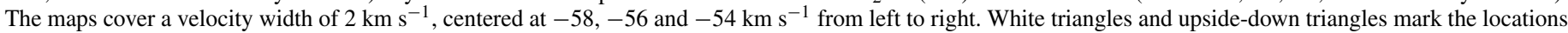

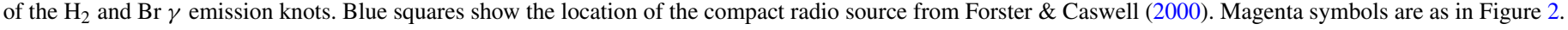
(A color version of this figure is available in the online journal.)

Table 1

Emission Features Detected in the Near-infrared ARC/TripleSpec Spectra, Numbered as in Figure 2

\begin{tabular}{lccccr}
\hline \hline Index & $\alpha(\mathrm{J} 2000)$ & $\delta(\mathrm{J} 2000)$ & Line & $\begin{array}{c}V \\
\left(\mathrm{~km} \mathrm{~s}^{-1}\right)\end{array}$ & $\begin{array}{c}\text { Line Flux } \\
\left(\times 10^{-16} \mathrm{erg} \mathrm{s}^{-1} \mathrm{~cm}^{-2}\right)\end{array}$ \\
\hline 1 & $17: 44: 40.107$ & $-29: 28: 16.77$ & $\mathrm{Br} \gamma$ & $-25 \pm 4$ & $19.12 \pm 0.02$ \\
2 & $17: 44: 40.163$ & $-29: 28: 13.75$ & $\mathrm{H}_{2}$ & $-34 \pm 12$ & $6.41 \pm 0.02$ \\
3 & $17: 44: 40.462$ & $-29: 28: 14.12$ & $\mathrm{H}_{2}$ & $-39 \pm 9$ & $11.85 \pm 0.02$ \\
4 & $17: 44: 40.469$ & $-29: 28: 13.07$ & $\mathrm{H}_{2}$ & $-66 \pm 27$ & $13.77 \pm 0.04$ \\
5 & $17: 44: 40.532$ & $-29: 28: 12.05$ & $\mathrm{H}_{2}$ & $-47 \pm 15$ & $9.11 \pm 0.03$ \\
\hline
\end{tabular}

at $2.12 \mu \mathrm{m}$ in two clusters approximately $5^{\prime \prime}(0.2 \mathrm{pc})$ apart. The eastern cluster contains three knots, with Gaussian-fitted $v_{\text {LSR }}$ of $-30,-50$ and $-70 \mathrm{~km} \mathrm{~s}^{-1}$; the western knot has a velocity of $-40 \mathrm{~km} \mathrm{~s}^{-1}$. The lines are unresolved at the TripleSpec spectral resolution.

Near the western $\mathrm{H}_{2}$ knot we find a site of $\mathrm{Br} \gamma$ emission at $-25 \mathrm{~km} \mathrm{~s}^{-1}$. Its proximity to the radio source $\left(1^{\prime \prime}\right)$ appears consistent with the presence of a hyper- or ultra-compact H II region; the velocity discrepancy may be the result of dynamical interaction between UCH II region and the host cloud.

Several emission features are detected along the slits away from the EGO. Their association with the region is unclear, and we do not include these further in our analysis. Properties of the detected emission features are summarized in Table 1. As the slit positions do not cover the extent of the IR emission, the detected knots may extend beyond the slit boundaries. Further knots may be found given better coverage.

\subsection{SMA $280 \mathrm{GHz}$ Data}

The $280 \mathrm{GHz}$ SMA data show two sources in the continuum bands associated with the EGO. Figure 3 shows the location of these sources with respect to the IR emission region.

The brightest, MM1, is located $\sim 6^{\prime \prime}(0.25 \mathrm{pc})$ south from the peak of the $4.5 \mu \mathrm{m}$ emission, coincident with a $6.7 \mathrm{GHz}$ maser (Caswell et al. 2010). It measures $5^{\prime \prime} .5 \times 5^{\prime \prime}$ in size $(0.2 \times 0.2 \mathrm{pc})$, measured to the $5 \sigma$ contour, and is elongated in the north-south direction (different from the beam elongation direction). At the location of the IR emission peak lies a second fainter source, MM2, also associated with a $6.7 \mathrm{GHz} \mathrm{CH}_{3} \mathrm{OH}$ maser. Measuring $3^{\prime \prime} \times 3^{\prime \prime}$ in size $(0.12 \times 0.12 \mathrm{pc})$, MM2 shows a similar elongation along the $\mathrm{N}-\mathrm{S}$ axis. Neither core appears associated with the radio source, suggesting the presence of at least three distinct star-forming sites. The integrated continuum fluxes measured within the $1 \sigma$ contours are $1073 \mathrm{mJy}$ for MM1 and $623 \mathrm{mJy}$ for MM2. We assumed uncertainties on these values of $15 \%$, dominated by the flux calibration accuracy.

In addition, the SMA data show $\mathrm{N}_{2} \mathrm{H}^{+}$line emission near the brighter core, with $v_{\mathrm{LSR}}$ ranging from -58 to $-54 \mathrm{~km} \mathrm{~s}^{-1}$. Channel maps and continuum data are shown in Figure 3.

\section{DISCUSSION}

\subsection{Distance to G359.44-0.102}

Dynamics of Sgr C are complex, with evidence of strong velocity gradients (Liszt \& Spiker 1995) and numerous absorption components in H I spectra (Lang et al. 2010). The velocity of the near $3 \mathrm{kpc}$ arm at a distance of $5.5 \mathrm{kpc}$ is very similar to the Sgr C systemic value at $l=-0.5$ (Oka et al. 1998), causing potential confusion; we note that Green et al. (2009) place the $\mathrm{CH}_{3} \mathrm{OH}$ masers in the $3 \mathrm{kpc}$ arm.

Three millimeter spectra from the Mopra CMZ survey (Jones et al. 2012) were extracted in a 40" aperture (the Mopra beam size) centered on the EGO for $\mathrm{HCO}^{+}(1-0)$ at $89.19 \mathrm{GHz}$, $\mathrm{H}^{13} \mathrm{CO}^{+}(1-0)$ at $86.75 \mathrm{GHz}$ and $\mathrm{SiO}(2-1)$ at $86.85 \mathrm{GHz}$ (Figure 4; the poor baseline calibration in weaker species is discussed by Jones et al. 2012). We show integrated velocity maps ( -45 to $-75 \mathrm{~km} \mathrm{~s}^{-1}$ ) alongside.

The spectra show strong emission peaks near $-54 \mathrm{~km} \mathrm{~s}^{-1}$ with fitted peak velocities consistent to within $1 \mathrm{~km} \mathrm{~s}^{-1}$. 

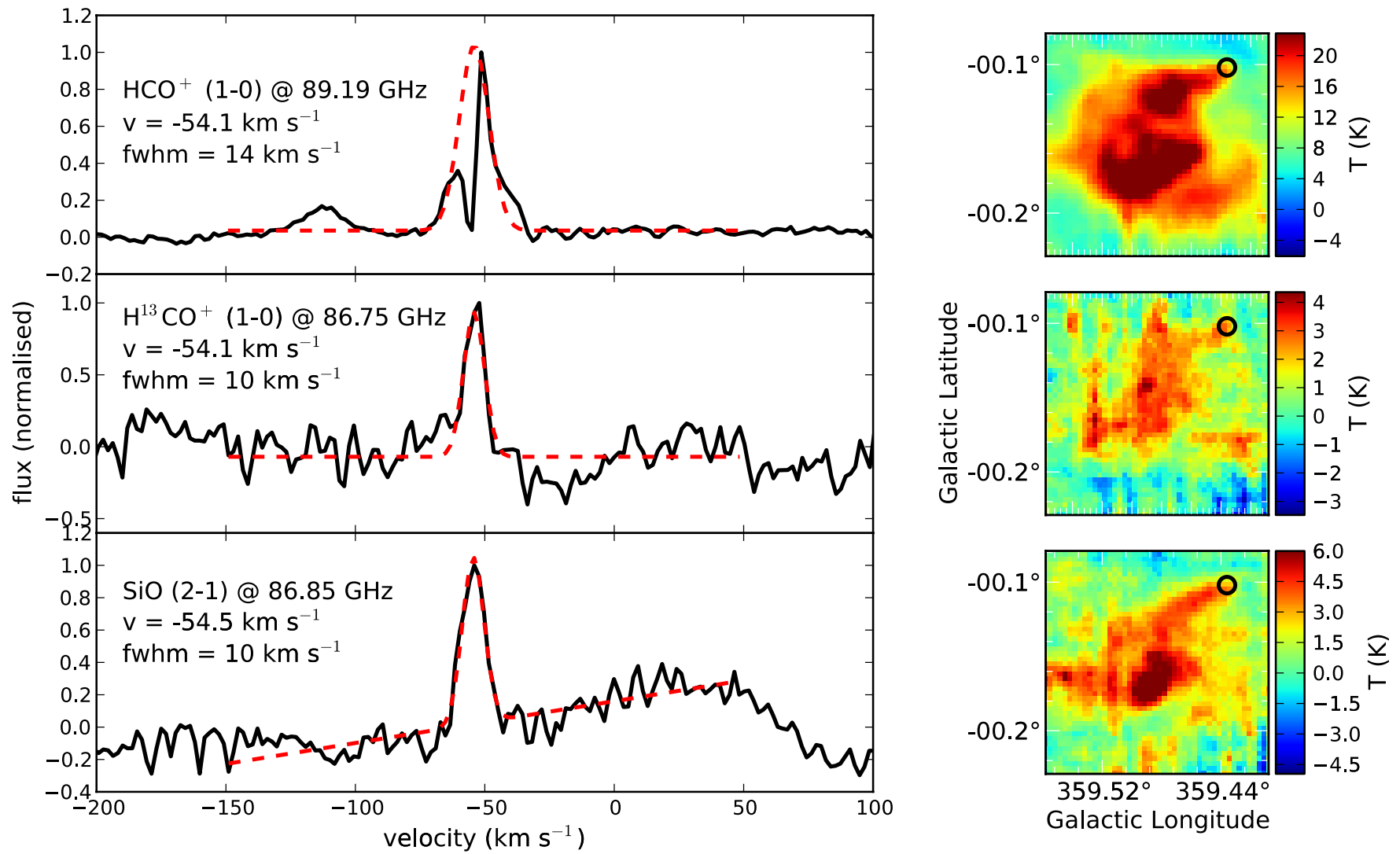

Figure 4. Mopra CMZ survey spectra (left), with (right) the corresponding integrated velocity map of the Sgr C cloud. The maps were integrated from -45 to $-75 \mathrm{~km} \mathrm{~s}^{-1}$, and cover the region of Figure 1(R). Spectra are extracted in a 40" aperture centered on G359.44-0.102 (see black circles) and spatially averaged. Black lines show the data, red dotted lines are the best-fit Gaussians. Errors on fit parameters are $<1 \mathrm{~km} \mathrm{~s}^{-1}$.

(A color version of this figure is available in the online journal.)

Linewidth measurements indicate full widths at half maximum of $>10 \mathrm{~km} \mathrm{~s}^{-1}$. Such broad linewidths are highly characteristic of CMZ clouds (Oka et al. 1998; Shetty et al. 2012); observed velocities for disk clouds are $\leqslant 5 \mathrm{~km} \mathrm{~s}^{-1}$ (Shetty et al. 2012).

The SiO (2-1) integrated velocity map shows widespread emission throughout the cloud. Extended $\mathrm{SiO}$ emission is a well-documented feature of CMZ clouds (Martin-Pintado et al. 1997; Riquelme et al. 2010); in the disk SiO emission is typically observed in outflow shocks locally.

These observed characteristics suggest that the EGO and its host cloud are located at the GC distance. Some uncertainty does however remain, the masses calculated in the following sections would in this case be reduced by $40 \%$.

\subsection{Physical Conditions in G359.44-0.102}

Integrated flux measurements from the SMA continuum data were used to estimate masses of the protostellar cores in the EGO using the following relation (Hildebrand 1983):

$$
M=\frac{g S_{\lambda} D^{2}}{\kappa_{\lambda} B_{\lambda}\left(T_{d}\right)},
$$

where $g$ is the gas-to-dust conversion ratio, $S_{\lambda}$ is the integrated flux at the observing wavelength $\lambda, D$ is the distance to the source, $\kappa_{\lambda}$ the dust mass opacity coefficient at $\lambda$, and $B_{\lambda}\left(T_{d}\right)$ is the blackbody flux for a dust temperature $T_{d}$ evaluated at $\lambda$. From the Hi-GAL-derived temperature map (Figure 1) we see that the EGO lies near the coldest part of the cloud at a temperature of $\sim 20 \mathrm{~K}$. We assume $g$ of 76 (Draine 2011, chap.
23), calculated using metallicity $Z_{\mathrm{GC}}=2 Z_{\odot}$ (Launhardt et al. 2002 ), and $D$ of $8.5 \mathrm{kpc}$.

For the dust opacity coefficient $\kappa$ we log-interpolate the data in Table 1 of Ossenkopf \& Henning (1994). For a gas density of $10^{6} \mathrm{~cm}^{-3}$ and grains with thin ice mantles, we find $\kappa_{1.06}$ of $1.25 \mathrm{~cm}^{2} \mathrm{~g}^{-1}$ for the SMA frequency of $280 \mathrm{GHz}$.

For the brighter continuum source MM1 the integrated flux yields a mass of $668 M_{\odot}$. Source MM2 has a mass of $380 M_{\odot}$. These values are averages of the masses calculated from the individual sideband fluxes. We estimate the combined uncertainty of these mass estimates to be a factor of $2-4$, based on estimates on uncertainty in flux calibration, $T_{d}, \kappa$ and $g$.

Similarly, peak column densities were computed using

$$
N\left(\mathrm{H}_{2}\right)^{\text {peak }}=\frac{S_{\lambda} g}{B_{\lambda}\left(T_{d}\right) \Omega \kappa_{\lambda} \mu m_{\mathrm{H}}},
$$

where $\Omega$ is the beam solid angle, $\mu$ is the mean molecular weight of the interstellar medium (assumed to be 2.8), $m_{\mathrm{H}}$ the mass of a hydrogen atom, and $g, S_{\lambda}$ and $B_{\lambda}\left(T_{d}\right)$ as defined above. Using the peak measured fluxes and taking a $2^{\prime \prime}$ SMA beam, we find peak column density estimates of $2 \times 10^{24} \mathrm{~cm}^{-2}$ and $1.1 \times 10^{24} \mathrm{~cm}^{-2}$ for sources MM1 and MM2, respectively.

\subsection{Evidence for Star Formation in G359.44-0.102}

The observational characteristics and derived properties of G359.44-0.102 are similar to those of other EGOs reported in the literature. In a sample of 28 objects, Cyganowski et al. (2009) found 64\% of EGOs to be associated with $6.7 \mathrm{GHz}$ Class 
II $\mathrm{CH}_{3} \mathrm{OH}$ maser emission, and of those $89 \%$ additionally have $44 \mathrm{GHz}$ Class I masers. Using millimeter and radio observations, Cyganowski et al. (2011a, 2011b, 2013) demonstrate that EGOs are reliable markers of massive star formation, often harboring multiple star-forming cores with strong active outflows.

The SMA continuum data show the presence of two star-forming cores, each carrying several $100 M_{\odot}$ in mass with high column densities, alongside the $\mathrm{UCH}$ II region proposed by Forster \& Caswell (2000). There is no evidence of significant dust heating toward these sources, nor of free-free or $\mathrm{Br}$ $\gamma$ emission suggesting a UCH II region has begun to form. The two main sites of $\mathrm{H}_{2}$ emission are clustered near the millimeter cores (Figure 2), with velocities both blue- and redshifted with respect to the systemic value.

In the absence of additional line detections, we cannot establish the excitation mechanism of these lines; however, the properties and locations of the knots show compelling evidence for the presence of at least one massive outflow. The detected millimeter cores are in close proximity $(\leqslant 0.5 \mathrm{pc})$ to the compact radio source, indicating that the EGO hosts at least three distinct sites of massive star formation.

\subsection{G359.44-0.102 in a Quiescent Host Cloud}

The EGO is located at the tip of a dense molecular cloud seen in absorption against the bright IR background. Survey data from the ATLASGAL and Hi-GAL surveys give an insight into the nature of this cloud.

The cloud measures roughly $7^{\prime} \times 4^{\prime}$ in ATLASGAL maps, corresponding to $\sim 16 \times 9 \mathrm{pc}$ at the GC distance. The Hi-GAL temperature map suggests temperatures across the cloud lie in the range of $20-25 \pm 2 \mathrm{~K}$, with a minimum of $19 \mathrm{~K}$ coinciding with the peak of the submillimeter emission.

Source extraction from ATLASGAL data by Contreras et al. (2013) identify three sources within the cloud (AGAL359.437-00.102, AGAL359.474-00.152, AGAL359. 514-00.154; Figure 1), measuring $3 \times 1 \mathrm{pc}, 4 \times 2 \mathrm{pc}$ and $1 \times 0.6$ pc. Contreras et al. (2013) report integrated fluxes of $119 \mathrm{Jy}, 168 \mathrm{Jy}$ and $25 \mathrm{Jy}$. Assuming parameters for Equation (1) as listed above and finding $\kappa_{0.87}$ of $1.75 \mathrm{~cm}^{2} \mathrm{~g}^{-1}$, these values yield gas masses of $\sim 4, \sim 5$ and $\sim 0.8 \times 10^{4} M_{\odot}$. The total mass enclosed in the dense cloud is thus of the order of $10^{5} M_{\odot}$, consistent with previous estimates (Lis et al. 1991; Lis \& Carlstrom 1994). The mass contained in cores MM1 and MM $2\left(<10^{3} M_{\odot}\right)$ represents just a small fraction of this total mass.

The clump-averaged column densities of the ATLASGAL sources were calculated using the effective radii from the Contreras catalog, and found to be in the range of 1.5-3.5 $\times$ $10^{22} \mathrm{~cm}^{-2}$. These values indicate that the entire cloud shows favorable physical conditions for massive stars to form (Lada et al. 2012). Apart from the data presented here for the EGO in source AGAL359.437-00.102, however, we found no 8, 24 or $70 \mu \mathrm{m}$ sources in the cloud indicative of further star formation activity. No radio emission is observed and no $\mathrm{CH}_{3} \mathrm{OH}$ masers are reported in the literature. The EGO's location at the cloud tip is likely to be pertinent, with star formation perhaps accelerated at the interface of a cloud-cloud collision or by feedback from the $\mathrm{H}$ II region. A detailed study of the dynamics of the region is required to understand which mechanism is at work.

The cloud shares similarities with the massive compact cloud G0.253+0.016 (Lis et al. 1994; Longmore et al. 2012; Kauffmann et al. 2013). The latter has a similar mass (1-2 $\times$ $\left.10^{5} M_{\odot}\right)$ as the Sgr C cloud, albeit in an apparently smaller volume, yet shows no evidence of significant star formation activity. Immer et al. (2012) report the discovery of a further four compact $10^{5} M_{\odot}$ clouds in the CMZ that appear devoid of star formation.

At the other extreme, the Sgr B2 star-forming region is one of the most prolific and well-studied in the Galaxy. Using $1.1 \mathrm{~mm}$ maps from the BGPS, Bally et al. (2010) find a mass of $5 \times 10^{5} M_{\odot}$ and average $\mathrm{H}_{2}$ column densities of $>10^{24} \mathrm{~cm}^{-2}$ toward its main star-forming sites. Its chemistry is however far richer, many of its star formation sites being more evolved hot cores and UCH II regions.

Based on our current data, Sgr C lies between the CMZ star-forming extrema represented by the G0.253 and Sgr B2. Despite the new evidence of star formation presented here, the Sgr C cloud as a whole still appears to be quiescent compared with non-CMZ clouds showing similar physical conditions (Lada et al. 2010). This adds to the evidence presented by Longmore et al. (2013) and Kauffmann et al. (2013) for an anomalously low star formation rate in the CMZ. A more detailed study of Sgr C is clearly required to establish the region's global star formation properties.

\section{CONCLUSIONS}

We have presented new, archival and survey data of the molecular cloud thought to be associated with Sgr C. Our highresolution IR and millimeter data show two massive protostellar cores associated with an EGO alongside a previously detected hyper- or ultra-compact $\mathrm{H}$ il region, which appear to be driving at least one outflow. The absence of evidence for dust heating indicates that these cores are at an early evolutionary stage.

The cores are deeply embedded in the densest region of a $\sim 16 \times 9$ pc molecular cloud abutting the Sgr C H II region. We note that the measured velocities also allow this site to be associated with the $3 \mathrm{kpc}$ arm at $5.5 \mathrm{kpc}$, however linewidths and widespread $\mathrm{SiO}$ emission indicate a likely distance of $8.5 \mathrm{kpc}$. The cloud harbors $\sim 10^{5} M_{\odot}$ of gas and dust with typical column densities of $>10^{22} \mathrm{~cm}^{-2}$ and temperatures of 19-25 K. It shows no evidence of further star formation activity despite presenting suitable physical conditions.

The presence of three star-forming cores in the EGO contrasts with the absence of further star formation in the host molecular cloud, placing Sgr C at an interesting mid-point between the quiescent G0.253 and starburst-like Sgr B2. The driving and inhibiting forces at work in the $\mathrm{CMZ}$ clouds and the relevance of their location along the proposed 100 pc elliptical ring are fascinating open questions.

S.K. thanks Jens Kauffmann, Sergio Molinari, Cornelia Lang and Yanett Contreras for sharing and discussing data. The Apache Point Observatory $3.5 \mathrm{~m}$ telescope is owned and operated by the Astrophysical Research Consortium. The Submillimeter Array is a joint project between the Smithsonian Astrophysical Observatory and the Academia Sinica Institute of Astronomy and Astrophysics. The Mopra radio telescope is part of the ATNF, funded by the Commonwealth of Australia.

Facilities: Spitzer (IRAC, MIPS), ARC, Mopra, APEX, UKIRT

\section{REFERENCES}

Bally, J., Aguirre, J., Battersby, C., et al. 2010, ApJ, 721, 137 Battersby, C., Bally, J., Ginsburg, A., et al. 2011, A\&A, 535, A128 Caswell, J. L. 2009, PASA, 26, 454

Caswell, J. L., Fuller, G. A., Green, J. A., et al. 2010, MNRAS, 404, 1029 Chambers, E. T., Yusef-Zadeh, F., \& Roberts, D. 2011, ApJ, 733, 42 
Contreras, Y., Schuller, F., Urquhart, J. S., et al. 2013, A\&A, 549, A45

Cyganowski, C. J., Brogan, C. L., Hunter, T. R., \& Churchwell, E. 2009, ApJ, 702,1615

Cyganowski, C. J., Brogan, C. L., Hunter, T. R., \& Churchwell, E. 2011a, ApJ, 743,56

Cyganowski, C. J., Brogan, C. L., Hunter, T. R., Churchwell, E., \& Zhang, Q. 2011b, ApJ, 729, 124

Cyganowski, C. J., Koda, J., Rosolowsky, E., et al. 2013, ApJ, 764, 61

Cyganowski, C. J., Whitney, B. A., Holden, E., et al. 2008, AJ, 136, 2391

Davis, C. J., Gell, R., Khanzadyan, T., Smith, M. D., \& Jenness, T. 2010, A\&A, 511, A24

De Buizer, J., \& Vacca, W. 2010, AJ, 140, 196

Draine, B. T. 2011, Physics of the Interstellar and Intergalactic Medium (Princeton, NJ: Princeton Univ. Press)

Forster, J. R., \& Caswell, J. L. 2000, ApJ, 530, 371

Green, J. A., McClure-Griffiths, N. M., Caswell, J. L., et al. 2009, ApJL, 696, L156

Hildebrand, R. 1983, QJRAS, 24, 267

Immer, K., Menten, K. M., Schuller, F., \& Lis, D. C. 2012, A\&A, 548, A120

Jones, P. A., Burton, M. G., Cunningham, M. R., et al. 2012, MNRAS, 419, 2961

Kauffmann, J., Pillai, T., \& Zhang, Q. 2013, ApJL, 765, L35

Lada, C. J., Forbrich, J., Lombardi, M., \& Alves, J. a. F. 2012, ApJ, 745,190

Lada, C. J., Lombardi, M., \& Alves, J. a. F. 2010, ApJ, 724, 687

Lang, C. C., Goss, W. M., Cyganowski, C., \& Clubb, K. I. 2010, ApJS, 191,275
Launhardt, R., Zylka, R., \& Mezger, P. G. 2002, A\&A, 384, 112

Lis, D., Carlstrom, J., \& Keene, J. 1991, ApJ, 380, 429

Lis, D. C., \& Carlstrom, J. E. 1994, ApJ, 424, 189

Lis, D. C., Menten, K. M., Serabyn, E., \& Zylka, R. 1994, ApJL, 423, L39

Liszt, H., \& Spiker, R. 1995, ApJS, 98, 259

Longmore, S. N., Bally, J., \& Testi, L. 2013, MNRAS, 429, 987

Longmore, S. N., Rathborne, J., Bastian, N., et al. 2012, ApJ, 746, 117

Martin-Pintado, J., de Vicente, P., Fuente, A., \& Planesas, P. 1997, ApJL, 482, L45

Molinari, S., Bally, J., Noriega-Crespo, A., et al. 2011, ApJL, 735, L33

Molinari, S., Swinyard, B., Bally, J., et al. 2010, PASP, 122, 314

Morris, M., \& Serabyn, E. 1996, ARA\&A, 34, 645

Odenwald, S., \& Fazio, G. 1984, ApJ, 283, 601

Oka, T., Hasegawa, T., Hayashi, M., Handa, T., \& Sakamoto, S. 1998, ApJ, 493, 730

Ossenkopf, V., \& Henning, T. 1994, A\&A, 291, 943

Reid, M. J., Menten, K. M., Zheng, X. W., et al. 2009, ApJ, 700, 137

Riquelme, D., Bronfman, L., Mauersberger, R., May, J., \& Wilson, T. L. 2010, A\&A, 523, A45

Schuller, F., Menten, K. M., Contreras, Y., et al. 2009, A\&A, 504, 415

Shetty, R., Beaumont, C. N., Burton, M. G., Kelly, B. C., \& Klessen, R. S. 2012, MNRAS, 425, 720

Stolovy, S., Ramirez, S., Arendt, R. G., et al. 2006, JPhCS, 54, 176

Varricatt, W. P., Davis, C. J., Ramsay, S., \& Todd, S. P. 2010, MNRAS, 404, 661

Yusef-Zadeh, F., Hewitt, J. W., Arendt, R. G., et al. 2009, ApJ, 702, 178 